\title{
Robustness and versatility of a nonlinear interdependence method for directional coupling detection from spike trains
}

\author{
Irene Malvestio, ${ }^{1,2,3, *}$ Thomas Kreuz, ${ }^{3}$ and Ralph G. Andrzejak ${ }^{1,4}$ \\ ${ }^{1}$ Department of Information and Communication Technologies, Universitat Pompeu Fabra, 08018 Barcelona, Spain \\ ${ }^{2}$ Department of Physics and Astronomy, University of Florence, 50119 Sesto Fiorentino, Italy \\ ${ }^{3}$ Institute for Complex Systems, CNR, 50119 Sesto Fiorentino, Italy \\ ${ }^{4}$ Institute for Bioengineering of Catalonia (IBEC), The Barcelona Institute of Science and Technology, 08028 Barcelona, Spain
}

(Received 26 April 2017; published 3 August 2017)

\begin{abstract}
The detection of directional couplings between dynamics based on measured spike trains is a crucial problem in the understanding of many different systems. In particular, in neuroscience it is important to assess the connectivity between neurons. One of the approaches that can estimate directional coupling from the analysis of point processes is the nonlinear interdependence measure $L$. Although its efficacy has already been demonstrated, it still needs to be tested under more challenging and realistic conditions prior to an application to real data. Thus, in this paper we use the Hindmarsh-Rose model system to test the method in the presence of noise and for different spiking regimes. We also examine the influence of different parameters and spike train distances. Our results show that the measure $L$ is versatile and robust to various types of noise, and thus suitable for application to experimental data.
\end{abstract}

DOI: 10.1103/PhysRevE.96.022203

\section{INTRODUCTION}

The detection of a dependence between the dynamics from experimental signals is very important for the understanding of a wide variety of systems. For instance, one of the fundamental problems in the study of brain dynamics is the reconstruction of networks from some measured signals [1]. These signals can be at the macroscopic level, including the electroencephalogram (EEG) and the magnetoencephalogram (MEG) [2-4]. In this work, we are interested in the neuronal level, testing a method applicable to recordings of spiking activity of individual neurons $[5,6]$.

Many different approaches have been developed for the detection of the driver-response relationship between two point processes. The first methods were based on cross-correlation $[7,8]$. Other methods are information-theory-based $[9,10]$, and they evaluate, for example, information transfer [11] or the transfer of entropy [12]. Granger causality assesses coupling by evaluating the ability of one spike train to forecast patterns in the other one $[13,14]$. There are methods based on maximum likelihood [15], and other methods are aimed at reconstructing the network structure of many neurons, with the assumption that the underlying node dynamics are known [16-18].

A different class of approaches to detect couplings is based on the asymmetric state similarity criterion [19]. These measures of nonlinear interdependence [19-22] quantify the likelihood that similar states of the response are mapped to similar states of the driver. Later the evaluation of the coupling strength was significantly improved by means of a rank-based statistics [23]. This measure, called $L$, was applied not only in neuroscience [24-26] but also in other fields; see, e.g., Ref. [27]. While initial applications were restricted to time-continuous signals, $L$ was subsequently extended to the study of point processes and also to the combination of point processes and flows [28]. The original method for time-continuous signals is based on a reconstruction of the signals in an embedding space [29], and similarity is assessed

\footnotetext{
*irene.malvestio@upf.edu
}

by the Euclidean distance in that space [23]. Instead, in the application to point processes, the similarity between different windows of the signals is quantified by a spike train distance [30]. The method is modular, meaning that different methods for estimating spike train similarity can be used in order to focus on specific properties of the signals, and also different nonlinear interdependence statistics can be applied.

In the paper in which the measure $L$ was first adapted to point processes [28], the capability of the method to detect unidirectional coupling was demonstrated on two model systems, namely the Lorenz dynamics and Hindmarsh-Rose model neurons. However, only one spike train distance was used, and the analysis was performed under rather simplified conditions. There was no noise, and only one possible regime of the Hindmarsh-Rose dynamics was covered (irregular spiking in the driver and bursting in the response). In this paper, we go a decisive step further, and using Hindmarsh-Rose dynamics we test the method under more challenging conditions, a prerequisite for any application to real data. First, we analyze the robustness of the method against different types of noise that reflect real conditions. Subsequently, we investigate its behavior in more problematic Hindmarsh-Rose regimes, not only irregular spiking and bursting but also regimes close to periodicity and exhibiting generalized synchronization. We still use a model system because it allows us both to simulate realistic experimental conditions and to validate the results. Throughout the analysis, we test different spike train distances in order to understand their advantages and disadvantages.

In this paper, we first describe the asymmetric state similarity criterion and the formulation of the measure $L$ based on the rank statistic [23], then we review the definitions of the spike trains distances and the Hindmarsh-Rose neuronal dynamics. We investigate the performance of the measure $L$ in the presence of noise, obtained using different spike train distances. Subsequently, we apply our method to other regimes, e.g., close to periodicity and in generalized synchronization regimes. Finally, we describe how to optimally choose the parameters, we interpret the results obtained with different distances, and we give an outlook on possible 
extensions of the method suitable for work in more complex settings.

\section{METHODS}

\section{A. Asymmetric state similarity criterion}

The method that we use to detect the presence of a coupling is based on the asymmetric state similarity criterion [19]. We consider two unidirectionally coupled dynamics, the driving dynamics $X$ and the response dynamics $Y$. The criterion is based on the fact that as a consequence of the unidirectional coupling, similar states of $Y$ are likely to be mapped to similar states of $X$. At first glance this appears counterintuitive, because it may seem that similar states in the driver should be mapped to similar states in the response. To understand the general idea better, we describe a simple example, namely the interdependence between the weather (driving dynamics) and the behavior of a girl called Alice (response dynamics). We consider just two states for both driver and response, i.e., the weather is either rainy or sunny, and Alice either carries an umbrella or not. Whenever Alice goes out with an umbrella (similar states of Alice's dynamics), the weather is rainy (similar states of weather dynamics). But if it is rainy, Alice does not always behave in the same way. For example, she might stay at home, with no need to use an umbrella. Hence when the coupling is unidirectional, there is an asymmetry in the probability that similarity in one signal implies similarity in the other. This asymmetry is exactly what the asymmetric state similarity criterion uses to detect the direction of the coupling. The degree of the mapping between similar states was used to quantify the strength of the interaction between the driver and the response [23,31]. Importantly, if the dynamics are close to synchronization, there can be a strong bidirectional mapping between the similarity in the two signals, without a pronounced asymmetry in the two directions. The strong state similarity would be evidence of high coupling, and the lack of asymmetry would weaken directionality detection [32].

We now describe how $L$ was used in Ref. [28] to apply this principle to spike train signals. First of all, it is necessary to have two signals $x(t)$ and $y(t)$ measured simultaneously from $X$ and $Y$, respectively. We assume that the two systems are both stationary. To make use of the asymmetric state similarity criterion, we need to define the states of the dynamics. In the case of time-continuous signals, the states are obtained by the state space reconstruction of the signals. They contain information about the present and recent past amplitude of the signal [22]. For spike trains, the analogous situation is to consider short windows. To obtain pairs of isochronous windows, we cut the signals in short pieces.

After defining the states of the dynamics, we need to assess the similarity between different states of the same signal, across different times. For this purpose, we calculate a matrix with the value $d_{i j}^{X}\left(d_{i j}^{Y}\right)$ of a chosen spike train distance (see Sec. II C) between all pairs of windows $i$ and $j$ ( $i, j=1, \ldots, N_{w}$, where $N_{w}$ is the total number of windows) of the signals $X(Y)$.

\section{B. Nonlinear interdependence measure}

Here we describe the algorithm to calculate the nonlinear interdependence measure $L$ proposed by Chicharro and
Andrzejak [23]. This measure evaluates the asymmetric state similarity criterion by quantifying the degree to which the similarity between states of one dynamics is mapped to the similarity between states of the other dynamics. It was already widely applied to time-continuous real-world signals, for example in the study of EEG [24-26], and also in contexts different from neuroscience [27].

In the previous section, we defined $d_{i j}^{X}$. Here we are interested in comparing the similarity between windows relative to the similarity between other pairs of windows. Therefore, we consider ranks of distances instead of exact values of distances. For every window $i$ of the $Y$ dynamics, $i=1, \ldots, N_{w}$, we define the quantity $r_{i l}^{Y}$, which is the index of the window with rank $l$ when sorted by the distance $d_{i j}^{Y}, j=1, \ldots, N_{w}$. We also define $g_{i, m}^{X}$ as the rank of the distance $d_{i m}^{X}$ in the sorted ascending list of the distances $d_{i j}^{X}$, $j=1, \ldots, N_{w}$. This allows us to calculate the $Y$-conditioned mean rank:

$$
G_{i}^{k}(X \mid Y)=\frac{1}{k} \sum_{l=1}^{k} g_{i, r_{i, l}^{Y}}^{X} .
$$

This quantity reflects the degree to which the first $k$ closest neighbors of the window $i$ in $Y$ are mapped to close neighbors of the same window in $X$. To estimate the interdependence between the dynamics, we take an average across all windows of a normalized value for $G_{i}^{k}(X \mid Y)$. In this way, we obtain $L(X \mid Y)[23]$ :

$$
L(X \mid Y)=\frac{1}{N_{w}} \sum_{i=1}^{N_{w}} \frac{G(X)-G_{i}^{k}(X \mid Y)}{G(X)-G^{k}(X)},
$$

where $G(X)=\frac{N_{w}}{2}$ is the mean rank expected for independent dynamics, while $G^{k}(X)=\frac{k+1}{2}$ is the minimal mean rank. The measure $L(X \mid Y)$ has the expected value 0 for independent dynamics. When the coupling strength from $X$ to $Y$ increases, $L(X \mid Y)$ increases as well. The maximum possible value is $L(X \mid Y)=1$, reached when $X$ and $Y$ are equal or so similar that their matrices of ranks of distances coincide. The measure $L$ is designed such that $L(X \mid Y)$ detects the coupling from $X$ to $Y$. To quantify the level of coupling in the other direction, we use $L(Y \mid X)$, obtained by switching the role of $X$ and $Y$ in Eq. (2) and in all previous definitions. We use $\Delta L(X, Y)=$ $L(X \mid Y)-L(Y \mid X)$ to assess the direction of the coupling [23].

The modularity of the approach based on the asymmetric state similarity criterion allows one to choose a statistics different from $L$ to evaluate the criterion. In the context of time continuous signals, other nonlinear interdependence measures were proposed $[20,21,33]$. Here we consider only $L$ because it was shown [23] to be more sensitive and specific for directional couplings than previous approaches.

\section{Assessing similarities between states: Spike train distances}

Since it is not defined a priori which should be the proper "distance" between different windows of a spike train, we study different possible choices. In the past 20 years, many different measures of spike train similarity were developed [30]. Among the best known are the Victor-Purpura distance [34] and the van Rossum distance [35]. Both depend on a 


\section{(a)}

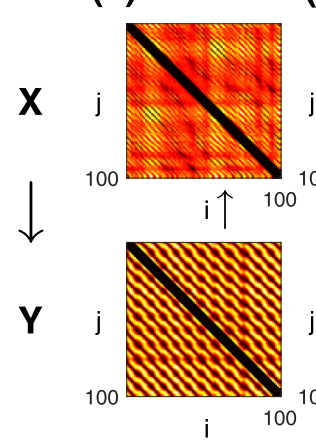

(b)

(c)
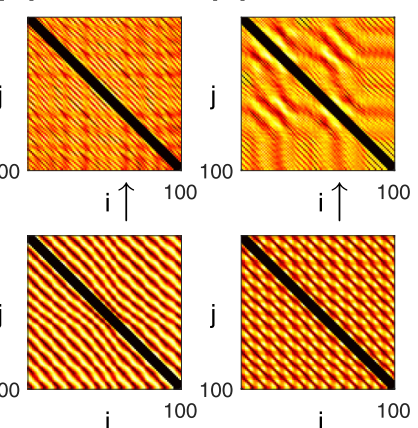

i 100

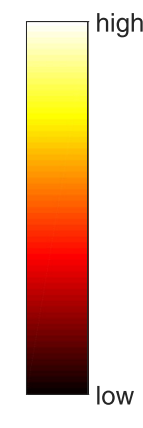

FIG. 1. Example of distance matrices $d_{i j}^{X}$ (first row) and $d_{i j}^{Y}$ (second row) calculated with three different spike trains distances: (a) van Rossum distance, (b) SPIKE distance, and (c) ISI distance. The signals are simulated from unidirectionally coupled Hindmarsh-Rose dynamics $X$ and $Y$ (driver and response, as indicated by the large arrow). The matrices are quite different even for the same signal. From the asymmetric state similarity criterion, we expect that the dark color in the $d_{i j}^{Y}$ matrices is likely to correspond to the dark color in the $d_{i j}^{X}$ matrices, as indicated by the direction of the small arrows. Elements close to the diagonal are not taken into account (see Sec. II C).

parameter that defines the time scale to which the method is mainly sensitive. This sensitivity determines the relative importance of rate versus exact timing of the spikes in computing the dissimilarities. Kreuz et al. developed the ISI distance [36] and the SPIKE distance [37], two timescale-independent, time-resolved distances that can be used to evaluate dissimilarity without the need to set a time scale.

These distances were applied not only in neuroscience [38-40], but also in other fields [41]. Recently, new generalizations of these distances were developed [42], called the A-ISI distance and the A-SPIKE distance. These generalized definitions add a notion of the relative importance of local differences compared to the global time scales. In particular, they start to gradually ignore differences between spike trains for interspike intervals (ISIs) that are smaller than a minimum relevant time scale.

In our work, we apply the van Rossum distance and the generalizations of ISI and SPIKE distances (see Appendix). In preanalysis, the Victor-Purpura distance yielded very similar results to the van Rossum distance, and thus it is omitted here. When the parameter of the van Rossum distance increases, the distance focuses more on spike rate than spike timing accuracy. On the one hand the parameter offers more flexibility, but on the other hand it is not obvious how to adjust it. Regarding the two parameter-free distances, we exploit the fact that the A-ISI distance focuses on differences of the rate, whereas the A-SPIKE distance focuses more on the exact timing, in order to gain complementary information about the system. In Fig. 1 we present an exemplary application of the three spike train distances to both the driver $X$ and the responder $Y$ of a unidirectionally coupled system. This example illustrates that the three distances give complementary information.

Segments of the signal of a fixed length $q$ are used as states of the dynamics for the calculation of the distance matrices. To obtain a finer sampling, we use overlapping windows, i.e., from one window to the next there is a step size $s$ with $s \leqslant$ $q$, which leads to the following segmentation of the signal: $[(i-1) s,(i-1) s+q]$ with $i=1, \ldots, N_{w}$. The total length of the point process is $Q$, and the total number of windows $N_{w}$ is obtained by rounding down $\frac{Q-q}{s}+1$. In every window, the time $\tau$ of a spike is relative to the beginning of the segment: $\tau=t-(i-1) s$, where $t$ is the time of the spike relative to the beginning of the whole signal. In the distance matrices, for every reference window we exclude the comparison with the $W=\frac{q}{s}-1$ overlapping neighboring windows, in analogy to the Theiler correction [43]. It is important to choose the parameters of the method properly. In general, a good choice for $q$ is the length of several mean interspike intervals, because a minimum number of spikes in every window is needed for a good evaluation of distances between windows. The parameter $s$ should be a good tradeoff between having more sensitive results (shorter $s$ ) and the required computation time (which is shorter for larger $s$ ).

\section{Simulated data: Hindmarsh-Rose neurons}

We use spike trains derived from coupled Hindmarsh-Rose model neurons ([44] and references therein) to test our method. This model is the same as that in [28], but here we consider more complicated settings. The system of equations is composed of a driver dynamics $X$,

$$
\begin{aligned}
& \dot{x}_{1}(t)=x_{2}(t)+3 x_{1}(t)^{2}-x_{1}(t)^{3}-x_{3}(t)+J_{x}, \\
& \dot{x}_{2}(t)=1-5 x_{1}(t)^{2}-x_{2}(t), \\
& \dot{x}_{3}(t)=0.0021\left\{-x_{3}(t)+4\left[x_{1}(t)+1.6\right]\right\},
\end{aligned}
$$

and a response dynamics $Y$,

$$
\begin{aligned}
\dot{y}_{1}(t)= & y_{2}(t)+3 y_{1}(t)^{2}-y_{1}(t)^{3}-y_{3}(t)+J_{y} \\
& +\epsilon Z\left(x_{1}(t)\right)\left[0.3-y_{1}(t)\right], \\
\dot{y}_{2}(t)= & 1-5 y_{1}(t)^{2}-y_{2}(t), \\
\dot{y}_{3}(t)= & 0.0021\left\{-y_{3}(t)+4\left[y_{1}(t)+1.6\right]\right\} .
\end{aligned}
$$

The coupling strength is $\epsilon$ and the coupling function is defined as

$$
\dot{Z}\left(x_{1}(t)\right)=\frac{Z_{\infty}\left(x_{1}(t)\right)-Z\left(x_{1}(t)\right)}{100\left[1-Z_{\infty}\left(x_{1}(t)\right)\right]}
$$

with

$$
Z_{\infty}\left(x_{1}(t)\right)= \begin{cases}\tanh \left[\mathrm{x}_{1}(\mathrm{t})+0.5\right] & \text { if } x_{1}(t)>-0.5 \\ 0 & \text { otherwise }\end{cases}
$$

The two dynamics are identical apart from the input currents $J_{x}$ and $J_{y}$, which moreover determine the dynamical regime of the signals. The type of coupling [Eqs. (5) and (6)] is a model for chemical synapses, whereas electrical synapses are described by a diffusive coupling [45]. The first components of Eqs. (3) and (4) represent the membrane potential of the neurons. In experiments, often one cannot measure the membrane potential but only the times of spikes. To simulate such experimental conditions, here we extract from these two time-continuous signals the spike times that compose the point processes $X$ and $Y$. The time of the spike is the instant in which the signal $x_{1}(t)$ crosses a threshold from below. As the threshold value, we chose $\Theta=0.6$. We call $t_{n}^{X}$ the time of the 

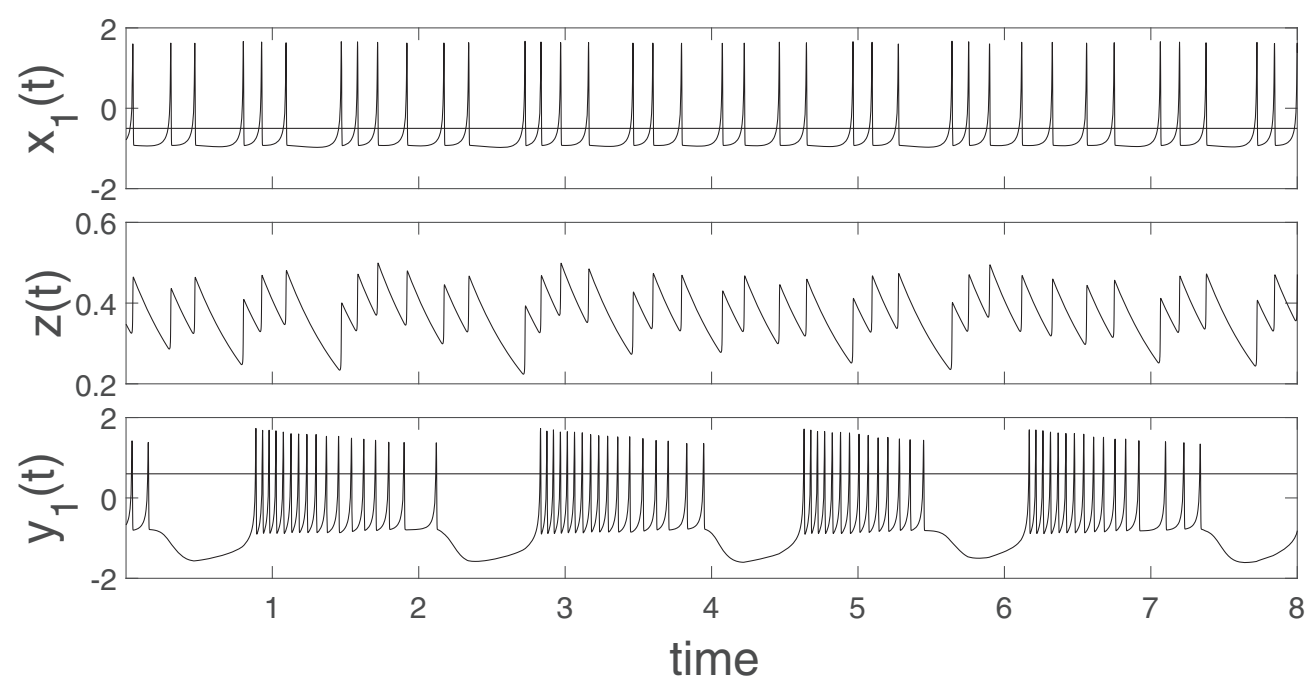

FIG. 2. Example signals for setting A: coupled Hindmarsh-Rose dynamics $\left[x_{1}(t), z(t), y_{1}(t)\right]$ with coupling strength $\epsilon=0.24, J_{x}=3.30$, and $J_{y}=3.28$. Horizontal lines represent the threshold for spike detection. The $x_{1}(t)$ signal is in an irregular spiking, the $y_{1}(t)$ signal in an irregular bursting regime.

$n$th spike, with $n=1, \ldots, N_{s}^{X}$, and $N_{s}^{X}$ being the total number of spikes in $X$. The times $t_{n}^{Y}$ are defined analogously.

The Hindmarsh-Rose equations were integrated with a fourth-order Runge-Kutta algorithm. The step size was 0.1 time units and the sampling step $\Delta t=0.2$ time units. We define $T=1000 \Delta t$ as the unit time interval. We started the dynamics with a random initial condition, and the first $500 T$ was discarded to exclude initial transients of the dynamics. The length of the spike trains was $Q=400 T$, and for the segmentation of the signals we used a window length of $q=T$ with an overlap of $s=0.2 T$. To avoid any type of in-sample optimization, this procedure was exactly the same as in the previous work [28].

Depending on the parameters $J_{x}$ and $J_{y}$, there can be different dynamical regimes of the Hindmarsh-Rose model, namely spiking or bursting regimes, and both can be periodic or aperiodic. In general, the resulting signals have different numbers of spikes, and furthermore the number of spikes in the response changes with increasing coupling. In our work, we analyze two settings. In setting A, we use the same regime as in [28] to test the robustness against noise for the different spike train distances. In setting B we consider different input currents and coupling values in order to validate the method for a range of different problematic dynamical regimes, such as dynamics close to periodicity and under generalized synchronization.

An example of the signals from setting A is shown in Fig. 2, with irregular spikes in the driving dynamics and irregular bursts in the response. The input currents are the same as in [28], $J_{x}=3.30$ and $J_{y}=3.28$. We computed $L$ for the uncoupled case plus $n_{\epsilon}=29$ coupling values $\epsilon$ equidistantly distributed on a logarithmic scale from 0.0006 to 0.24 . For each $\epsilon$, we run $n_{r}=20$ independent realizations of the dynamics. We say that the coupling and its direction for a specific value of $\epsilon$ are detected if the mean of the difference on the $n_{r}$ realizations $\langle\Delta L(X, Y)\rangle=\langle L(X \mid Y)-L(Y \mid X)\rangle$ is significantly larger than zero. To assess the significance, we performed a Wilcoxon signed rank test on $\langle\Delta L(X, Y)\rangle$. The level of significance was $\alpha=0.05 / n_{\epsilon}$, where we used the Bonferroni correction [46] to take into account the multiple testing of the $n_{\epsilon}$ values of the coupling. We use this setting to study the robustness of the measure $L$ to noise. We define the performance $\Psi$ to be the percentage of the $n_{\epsilon}$ coupling values for which we can significantly detect the coupling and its direction. To quantify the variability of $\Psi$, we ran three times a total of $n_{r}$ realizations, and we calculated the mean and the variance of the performances. When we compare the performance for the robustness to noise with different spike train distances, for the ISI distance and the SPIKE distance there is no need to choose a parameter. Instead, in the case of the van Rossum distance, we compute the performance $\Psi$ for a range of parameters and we select the one that leads to the best performance.

In setting B we consider different input currents $\left(J_{x}=3.28\right.$, $\left.J_{y}=3.60\right)$. We consider the uncoupled case plus $n_{\epsilon}=89$ coupling values $\epsilon$ equidistantly distributed on a logarithmic scale from $6 \times 10^{-6}$ to 1.8 . Here the driving dynamics is in an irregular bursting regime, while the response shows a variety of different behaviors. It is close to periodicity for very small coupling values [Fig. 3(a)], while it undergoes a bifurcation as the coupling increases, until it reaches an irregular bursting regime [Fig. 3(b)]. Finally, for the highest coupling values there are degenerated bursts [Fig. 3(c)]: the spikes in the bursts show a decrease in the amplitude from the beginning to the end of the burst, and the amplitudes of the last ones are so small that the spikes do not reach the threshold for detection any longer. The very irregular shape of the spikes makes them not so well-defined as in the other regimes, and even adjusting the threshold would not be effective. Therefore, as the coupling increases, the detected spikes preserve less and less of the information contained in the time-continuous signals.

There are two intervals of coupling values for which the driving and the response are in generalized synchronization [47]. The concept of generalized synchronization comes from the study of time-continuous signals. Two dynamics $X$ and $Y$ are in generalized synchronization when the trajectories $y(t)$ of $Y$ can be derived by a functional relationship from the corresponding trajectories $x(t)$ of $X$ [48]. In this way, 

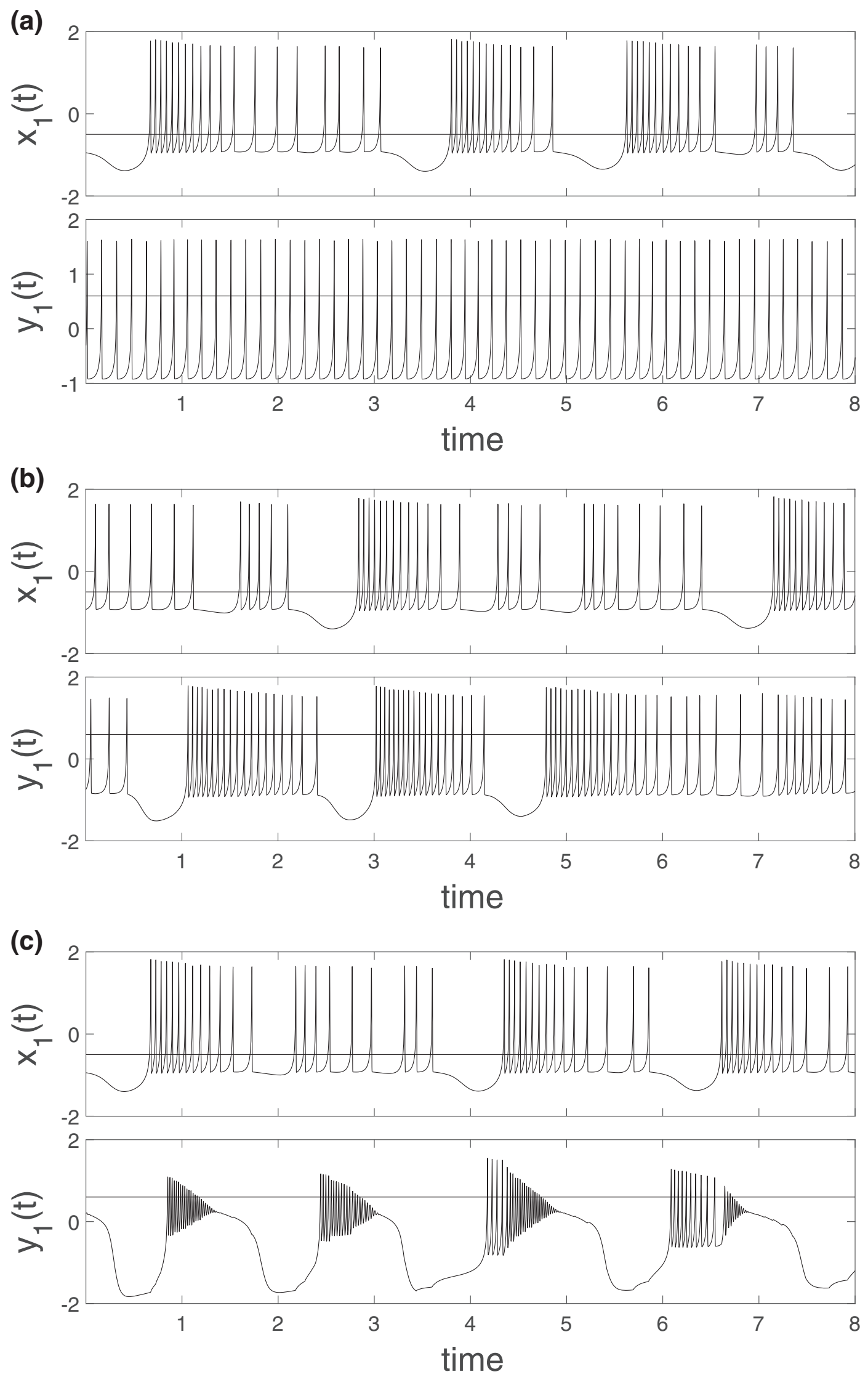

FIG. 3. Example signals for setting B: coupled Hindmarsh-Rose dynamics $\left[x_{1}(t), y_{1}(t)\right]$ with $J_{x}=3.28$ and $J_{y}=3.6$. In all the panels, the $x_{1}(t)$ signal is in an irregular bursting regime. The $y_{1}(t)$ signal is very close to periodicity in panel (a), where the coupling strength is $\epsilon=0.0101$, in an irregular bursting regime in panel (b), $\epsilon=0.1092$, and finally it presents degeneration of the bursts in panel (c), $\epsilon=1.0276$. 
$y(t)$ is not dependent anymore on its initial conditions. To check for which coupling values the dataset is in the regime of generalized synchronization, we use the auxiliary system approach [49], exploiting the fact that the spike trains that we study are extracted from time-continuous signals. For a realization of the driving dynamics $X$ [Eq. (3)], we generate two realizations of the response dynamics, $Y$ and $Y^{\prime}$, which obey the same Eqs. (4) but start from different initial conditions. We wait a long transient (50 000T) and we compute the average of the difference between the last $400 T$ of $y_{1}(t)$ and $y_{1}^{\prime}(t)$. If this distance is zero, that means that the $Y$ dynamics is independent of its initial condition, therefore generalized synchronization is established.

\section{E. Noise}

In this section, we introduce different noise types that we apply to the simulated signals of setting A. We select them in order to cover some of the principal disturbances that can affect data in the real world. The principal noise sources are due to the intrinsic stochasticity of the neurons [50,51], the uncertainty due to spike sorting, and other measurement noises [5].

At first we consider a noise type that we call unreliability noise. It represents the possibility of having false-positive and false-negative detections of spikes. We simulated this noise by randomly removing and inserting spikes. In the original point process $t_{n}^{X}$, with $1 \leqslant n \leqslant N_{s}^{X}$, we remove $0 \leqslant M \leqslant N_{s}^{X}$ spikes. At the same time, we randomly insert $M$ new spikes. The positions of the new spikes are uniformly distributed in the interval between 0 and the total recording time $Q$. This way, the new spike train $\widetilde{t}_{n}^{X}$ has the same number $N_{s}^{X}$ of spikes, while the interspike interval distribution is not maintained. We define the noise level $\gamma_{u}$ as the fraction of spikes that we remove and insert $M=\gamma_{u} N_{s}^{X}$. Analogous definitions hold for the $t_{n}^{Y}$ spike train.

The second type of noise is a shift in the spike times, the jitter noise. In this case, we shift every spike time of both $t_{n}^{X}$ and $t_{n}^{Y}$ by a different delay $\delta t$ extracted from a Gaussian distribution with zero mean and standard deviation $\sigma$. We choose $\sigma$ as a fraction $\gamma_{j}$ of the mean interspike interval of the time series $\mu_{\mathrm{ISI}}: \sigma=\gamma_{j} \mu_{\mathrm{ISI}}$. The noise level is quantified by $\gamma_{j}$.

The third type of noise reflects the fact that spike propagation depends on unreliable connections between neurons; for example, some spikes of two connected neurons may not be transmitted. We represent this effect by modifying a term in the equations, hence it is a dynamical noise that we call transmission noise. In the Hindmarsh-Rose Eqs. (3)-(6), the coupling between the neurons is mediated by the coupling function $Z\left(x_{1}(t)\right)$ [Eq. (5)], which is influenced by $x_{1}(t)$ only when $x_{1}(t)$ is higher than the threshold of -0.5 , i.e., in correspondence to a spike in $x_{1}$. We cancel the influence from single spikes of $X$ on $Y$, with some probability $\gamma_{t}$, which quantifies the noise level. Thus, for the whole time of the increase of the membrane potential due to a specific spike in $x_{1}$, we let the function $Z$ evolve as if the threshold was not crossed. It is important to notice that by ignoring spikes, we reduce the "effective" coupling strength.

\section{RESULTS}

In this section, we show the influence of different distances and parameters on the efficiency of $L$. First, we study the
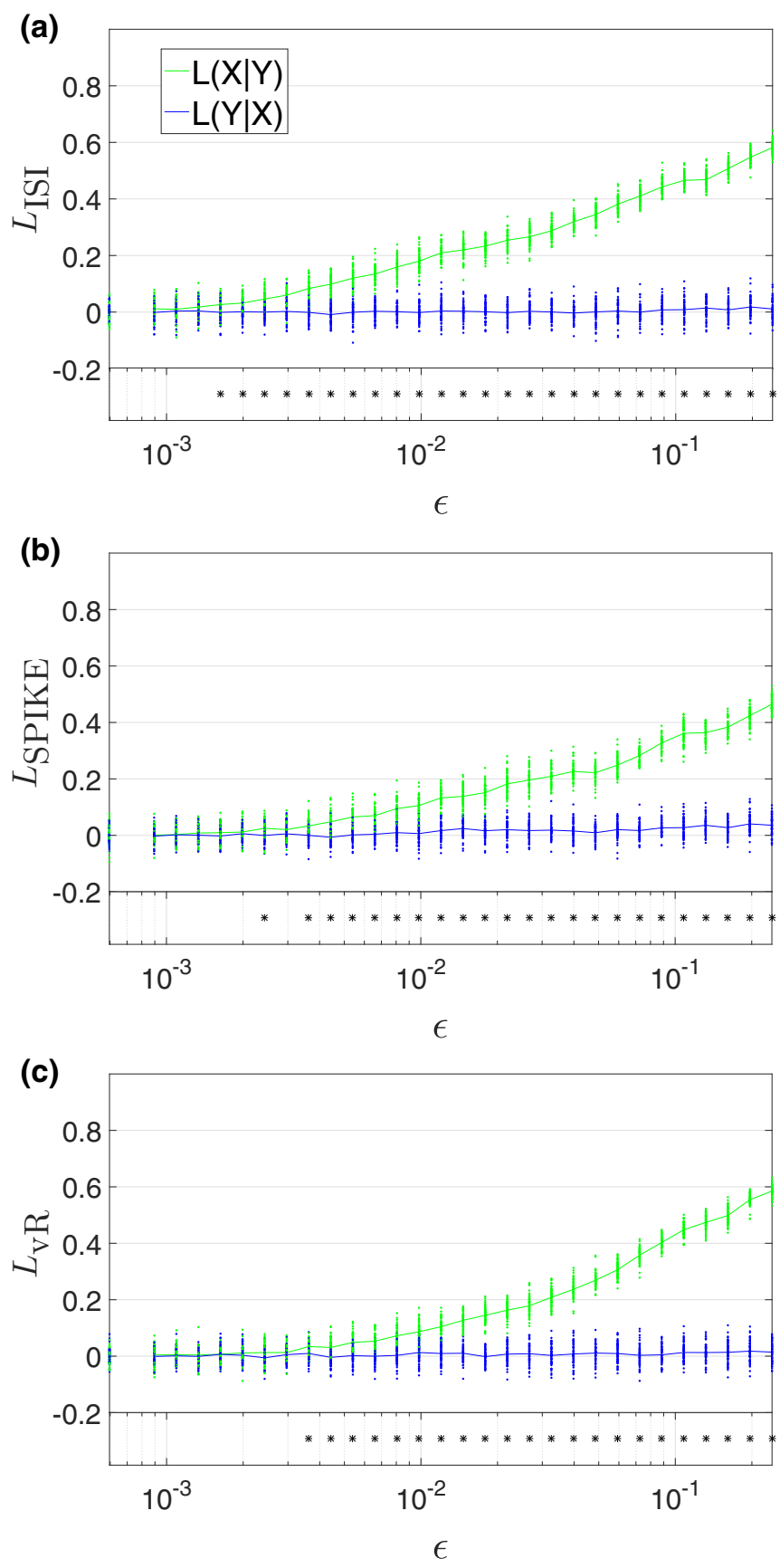

FIG. 4. Values of $L$ for increasing coupling strength $(k=1)$. From top to bottom, $L_{\mathrm{ISI}}, L_{\mathrm{SPIKE}}$, and $L_{\mathrm{vR}}$ (with the optimal parameter for significant coupling detection). For every coupling value, there are 20 independent realizations. Results for uncoupled dynamics $(\epsilon=0)$ are displayed on the ordinates. The crosses on the bottom of the plots mark the coupling values for which the detection of the coupling is significant. The measure $L_{\mathrm{ISI}}$ performs best, since it is able to detect even low couplings.

dependence on the coupling strength without noise. Subsequently, we investigate the robustness of $L$ to various kinds of noise. Finally, we test the versatility of this approach in different dynamical regimes.

We start our analysis with the noise-free case of setting A. In Fig. 4 we investigate the dependence of $L$ on the coupling 
(a)

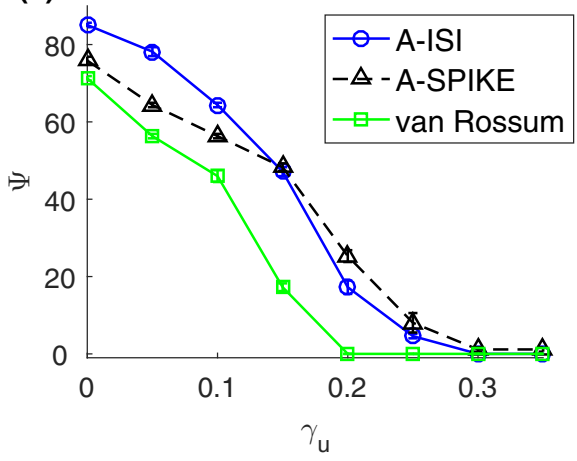

(d)

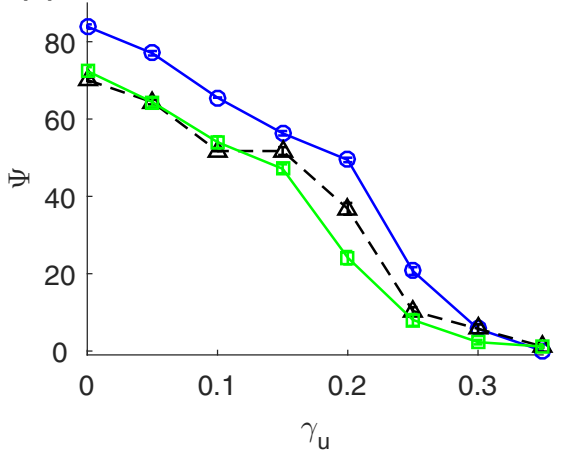

(b)

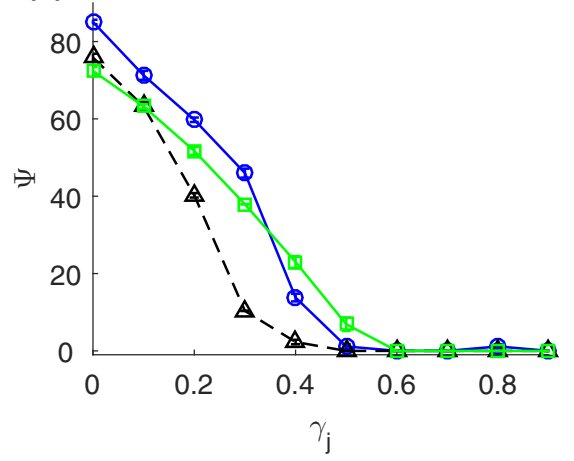

(e)

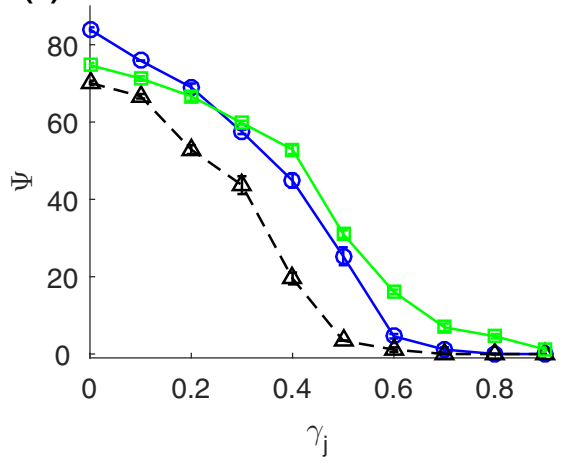

(c)

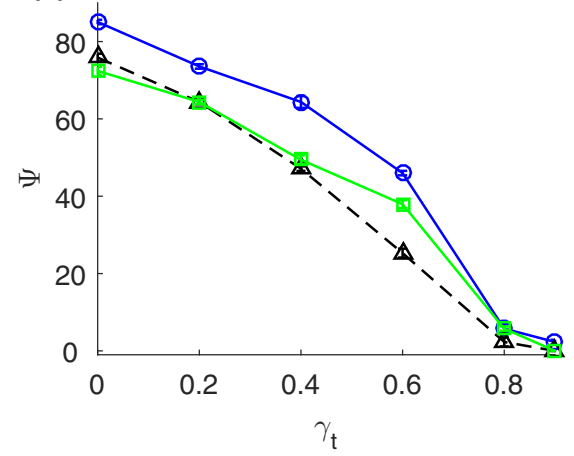

(f)

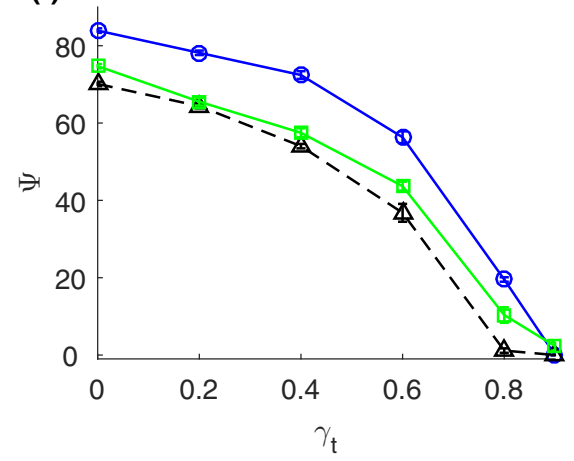

FIG. 5. Comparison of the performance $\Psi$ of the measure $L$ for different spike train distances in dependence on increasing levels of (a,d) unreliability, (b,e) jitter, and (c,f) transmission noise, in unit of the corresponding noise levels $\gamma_{u}, \gamma_{j}$ and $\gamma_{t}$. In the first row (a-c) we set $k=1$, in the second row ( $\mathrm{b}-\mathrm{d}) k=10$. The A-ISI distance performs best. The robustness to noise is improved by a higher number of nearest neighbors $k$.

strength for the A-ISI distance $\left(L_{\mathrm{ISI}}\right)$, the A-SPIKE distance ( $\left.L_{\text {SPIKE }}\right)$, and the van Rossum distance $\left(L_{\mathrm{vR}}\right)$. The measure $L_{\mathrm{ISI}}$ can distinguish the direction even for small $\epsilon$ and it also gives higher values of $L$. The measure $L_{\mathrm{vR}}$ instead, even with the parameter that optimizes the performance $\Psi$, performs worse and has lower values than $L_{\text {ISI }}$. The $L_{\text {SPIKE }}$ performs worst.

In Fig. 5 we continue to analyze setting $\mathrm{A}$, but now we investigate the effect of the different spike train distances on the robustness against noise of $L$. We also look at the influence of $k$ on the performance. In the presence of unreliability noise, generally $L_{\mathrm{ISI}}$ continues to perform best, even if for high levels of noise $L_{\text {SPIKE }}$ starts to perform better [Fig. 5(a)]. It is clear that selecting a high number of neighbors is very important for the robustness of the method, since the overall performance increases [Fig. 5(b)]. At the same time, for $k=10, L_{\mathrm{ISI}}$ performs best across the entire range of noise levels. For larger $k$ the value of $L$ decreases in general, but also the variance of $L$ across different realizations decreases because averaging over more neighbors gives more stable results. As a consequence, the direction detectability improves, in particular in the presence of noise. The measure $L_{\mathrm{vR}}$, even though its parameter is optimized separately for every noise level, performs worst. Regardless of the spike train distance, up to $35 \%$ of the spikes have to be removed and randomly reinserted in order to completely destroy the performance. Thus we can conclude that the measure $L$ is robust to unreliability noise.

For jitter noise [Figs. 5(c) and 5(d)] $L_{\mathrm{vR}}$ performs very well; in particular, it slightly outperforms $L_{\text {ISI }}$ for high levels of noise. Every spike has to be shifted by a value extracted from a Gaussian distribution of standard deviation $70 \%$ of the mean interspike interval in order to lead to zero performance. Accordingly, we can state that the method is robust also to jitter noise.

The measure $L_{\text {ISI }}$ performs best also with the addition of transmission noise [Figs. 5(e) and 5(f)]. Despite the optimization, $L_{\mathrm{vR}}$ performs worse than $L_{\mathrm{ISI}}$. The performance is zero only if $90 \%$ of the spikes of the driven dynamics are ignored by the coupling, hence the measure $L$ is robust also to transmission noise. We notice that in this case the performance stays high for high levels of noise and then it drops more suddenly at the end. The dependence on noise level is therefore different from that for the other noise types.

One interesting aspect of this analysis concerns the strong dependence of the van Rossum distance on the time-scale parameter (results not shown). Putting the focus on rate works better in the presence of high levels of jitter noise, whereas for high levels of unreliability noise it is preferable to focus on spike accuracy. This is intuitive and in agreement with the comparison of $L_{\mathrm{SPIKE}}$ and $L_{\mathrm{ISI}}$ in the presence of these two types of noise. However, this dependence of the optimal parameter on the type and level of noise renders the use of the van Rossum distance problematic for applications to realistic situations when all kinds of noise are present at the same time and the ground truth is unknown.

In setting $\mathrm{B}$, we focus on the adaptability of the measure $L$ to spike trains which reflect the different regimes of the underlying dynamical systems (Fig. 6). To avoid the complications caused by the time-scale parameter, we do not 


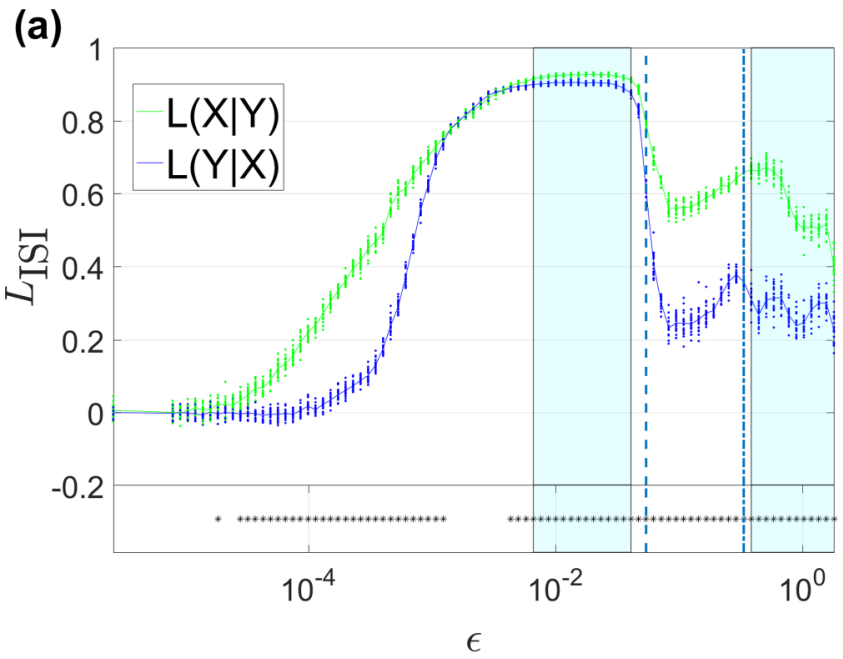

(b)

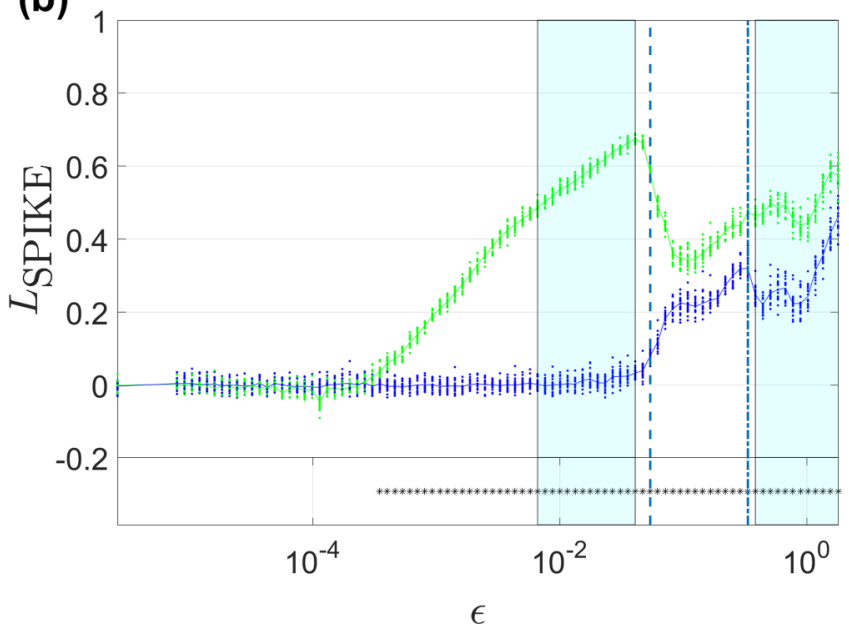

FIG. 6. Values of $L_{\mathrm{ISI}}$ and $L_{\mathrm{SPIKE}}$ in dependence on the coupling strength $\epsilon(k=10)$. Results for uncoupled dynamics $(\epsilon=0)$ are displayed on the ordinates. The crosses on the bottom of the plots mark the $\epsilon$ values for which the direction of the coupling is significantly detectable. The shaded areas correspond to the generalized synchronization regimes. The first dashed vertical line on the left distinguishes the regime close to periodicity [corresponding to small coupling values, see Fig. 3(a)] from the regime with bursts in the response [see Fig. 3(b)], and the second line marks the transition to the regime for which there is a strong degeneration of the bursts [see Fig. 3(c)].

consider the van Rossum distance any longer (and the same applies to the time-scale-dependent Victor-Purpura distance). From Fig. 6(a) we can see that $L_{\text {ISI }}$ is highly sensitive to small coupling values, even if in this regime the response is close to periodicity [compare Fig. 3(a)]. At the same time, $L_{\text {SPIKE }}$ cannot detect the very small coupling values [Fig. 6(b)]. When $\epsilon$ increases, the values of $L$ for both spike train distances increase, but their behavior differs. The directionality detection of $L_{\text {ISI }}$ deteriorates until the values of $L_{\text {ISI }}$ are very close to 1 and they almost coincide in both directions. The results of the auxiliary system approach in Fig. 7 confirm that the high values of $L_{\text {ISI }}$ correspond to coupling values close to and inside the first interval of the generalized synchronization regime. Surprisingly, for the corresponding coupling values,

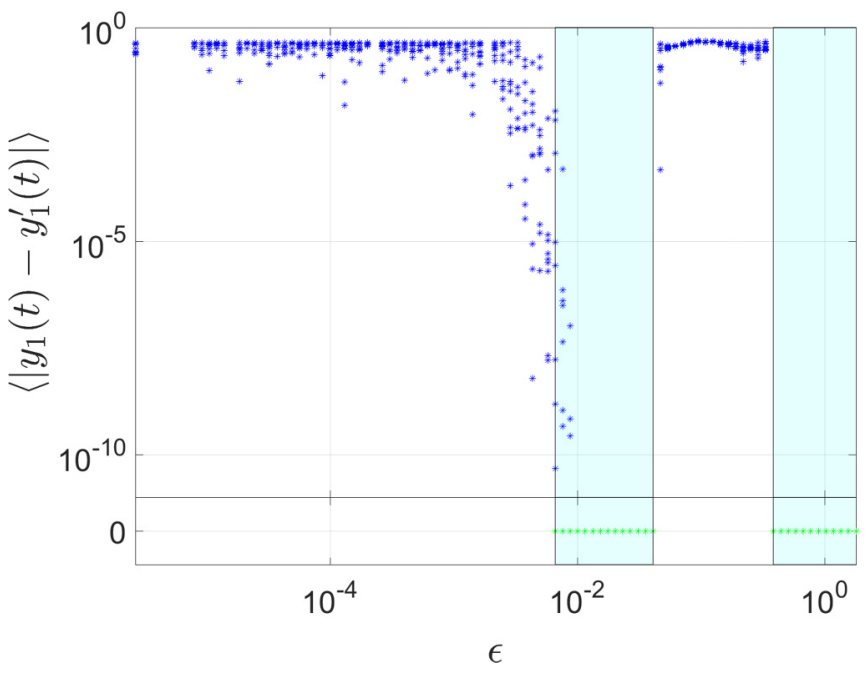

FIG. 7. Results of the auxiliary system approach applied to setting $\mathrm{B}$ of the Hindmarsh-Rose model. We plot the average of the absolute value of the difference between the last $400 T$ corresponding values of $y_{1}(t)$ and its copy $y_{1}^{\prime}(t)$, after discarding a transient of $10^{5} T$. For every coupling value there are five independent realizations, each of them represented by an asterisk. The crosses that correspond to values of exactly zero in at least one realization mark the regime of generalized synchronization. Results for uncoupled dynamics $(\epsilon=0)$ are displayed on the ordinates.

$L_{\text {SPIKE }}$ can perfectly detect both the presence and direction of the coupling.

The behavior of $L_{\text {ISI }}$ resembles the behavior of $L$ for time-continuous signals when the coupling is approaching a value large enough to induce generalized synchronization [23]. This behavior is well known in the study of timecontinuous signals [52]. As a consequence, a requirement for the applicability of directionality detection is that the coupling does not induce synchronization [32,53]. The high sensitivity of $L_{\text {ISI }}$ leads more easily to high values for the coupling without any sign of directionality when approaching the regime of generalized synchronization. In contrast, while being less sensitive, $L_{\text {SPIKE }}$ in the generalized synchronization regime can perfectly detect the direction of the coupling. There might be two reasons for these results. First, the rate compared to the exact timing is more easily affected by a small coupling. Second, the functional relation of generalized synchronization that connects the $y_{1}(t)$ and $x_{1}(t)$ signals of the Hindmarsh-Rose model can be very complicated, and it is possible in principle that it is easier to detect from spike rate instead of spike timing. A complete study of generalized synchronization between two Hindmarsh-Rose dynamics is beyond the scope of our study. Nevertheless, empirically we see that generalized synchronization expresses itself in different features.

After the first synchronization window, while both values $L_{\text {ISI }}(X \mid Y)$ and $L_{\text {SPIKE }}(X \mid Y)$ show a decrease, a distinction of the coupling direction is still possible (Fig. 6). These coupling values correspond to the change of regime in the response, from almost regular spiking to irregular bursting [compare Fig. 3(b)]. In this case, the values of $L$ increase also in the opposite direction $L(Y \mid X)$. We remind the reader 
that $L(Y \mid X)$ would indicate a coupling from $Y$ to $X$. For the highest coupling values, $L_{\mathrm{ISI}}$ decreases again in both directions [Fig. 6(a)]. Furthermore, there is another window of generalized synchronization. However, both $L_{\text {ISI }}$ and $L_{\text {SPIKE }}$ behave differently as compared to the first window. Here the corresponding signals show an additional effect, the degeneration of the bursts [see Fig. 3(c)]. The spikes contain less information about the original time-continuous signals. Moreover, spike density in the bursts is very high, and there are longer interburst periods. This bursting structure leads to smaller values of $L$ despite the higher coupling strength, compared to the previous regime close to periodicity.

\section{DISCUSSION}

The detection of directional interdependence between unknown dynamics from their signals is a nontrivial problem, and in the past few decades many different approaches to address this problem have been proposed. Before using a method to interpret real data, it is always important to first study its properties and to apply it to model systems. Thus in this paper we simulate realistic experimental conditions to validate a method for the detection of unidirectional couplings between point processes. This approach is based on the asymmetric state similarity criterion in the formulation of the nonlinear interdependence measure $L$ [23]. The capability of this method to detect coupling was already demonstrated in the Lorenz dynamics and in one setting of Hindmarsh-Rose dynamics [28]. Here we examine the robustness against various kinds of noise and the versatility of the approach in dealing with different dynamical regimes. Additionally, we take advantage of the modularity of the measure $L$ and test three spike train distances that are sensitive to different aspects of the dynamics.

Our results show that among the three distances that we used for the measure $L$, the A-ISI distance exhibits the highest sensitivity in detecting unidirectional coupling. While all three distances prove to be very robust in the presence of noise, again the measure $L_{\text {ISI }}$ performs best. The measure $L$ in general works for different dynamical regimes, including irregular spiking, irregular bursting, almost periodic motion, and under generalized synchronization. Interestingly, $L_{\text {ISI }}$ and $L_{\text {SPIKE }}$ catch different features of the coupling between spike trains, namely an interdependence based on the rate or on exact spike timing, respectively. Therefore, it is recommendable to apply both $L_{\text {ISI }}$ and $L_{\text {SPIKE }}$, because they provide complementary information and might therefore be sensitive to different coupling strengths.

In general, the values of $L$ are not monotonically increasing with an increase of the coupling value. As emphasized in [54], the effect of the coupling depends not only on the coupling strength but also on the energy of the individual dynamics. As a consequence, the estimate of the coupling is not independent from the structure of the data, for example in bursting or spiking regimes.

The choice of the parameters is important to obtain meaningful results. In particular, we have seen that a higher number of nearest neighbors $k$ is an advantage in the presence of noise. Nonetheless, no fine-tuning of $k$ is needed, and a value of approximately $0.5 \%$ of the total number of windows is a good choice in order to be less affected by noise. Another important parameter is the window length. If it is too long, it reduces the reliability of the measure $L$ in the sense that there will be too few windows to compare. If it is too short, the estimation of similarity is not reliable. To obtain robust results, we recommend a minimum of five spikes in each window. Furthermore, the relevant information about similarity can be contained in a complex structure, such as the bursts, which cannot be properly captured by very short windows.

It was shown in the previous study [28] and is further supported by our results that the measure $L$ for the detection of directional coupling between point processes performs well in the study of simulated spike trains derived from nonlinear deterministic dynamics. In the application to experimental data, it would be interesting to first use a measure that quantifies the determinism from the signal [55] in order to assess the level of nonrandomness present in the data. For example, if the spike trains are from two coupled stochastic point processes, the measure $L$ could have trouble with the detection of directionality, even if the presence of a coupling is still detectable. Another caveat is the possibility to have a small but significant difference in the values of $L$ in the two directions also for bidirectionally coupled dynamics [54]. As a consequence, in the interpretation of experimental results, more caution is necessary to assume directionality if $L(X \mid Y) \approx L(Y \mid X)$. An open question concerns the efficiency of the measure $L$ under these conditions and how to quantify the reliability of directionality detection.

Since different methods can be used to extract information from different features of the data, it is recommended not to restrict oneself to just one approach, such as nonlinear interdependence measures, but instead to use complementary techniques for directionality detection [8-15]. A comprehensive comparison between the different methods regarding their statistical requirements, their computational cost, and their abilities to provide complementary information on the dynamics under investigation will be the focus of future studies.

In this paper, we have considered only pairs of unidirectionally coupled dynamics. The next step is the analysis of multivariate data, with the perspective of network reconstruction. Making use of the modularity of the approach based on the measure $L$ can give complementary information about interdependence, and this may be useful to discriminate real from spurious or indirect connections. Moreover, while here we have focused on neuronal signals, the measure $L$ seems to be a very promising tool also for applications outside neuroscience, for example in climatology [56,57] or earthquake prediction [58].

In closing, we would like to point out that we provide the MATLAB source codes to calculate $L$ and the spike train distances used here [59].

\section{ACKNOWLEDGMENTS}

We acknowledge funding from the European Union Horizon 2020 research and innovation programme under the Marie Skłodowska-Curie Grant Agreement No. 642563 "Complex Oscillatory Systems: Modeling and Analysis" (COSMOS), (I.M., T.K., R.G.A.), and from the Volkswagen Foundation, the Spanish Ministry of Economy and Competitiveness Grant 
No. FIS2014-54177-R and the CERCA Programme of the Generalitat de Catalunya (R.G.A). We thank P. Laiou and E. Satuvuori for useful discussions. We thank E. Satuvuori and A. Font for technical support.

\section{APPENDIX: SPIKE TRAIN DISTANCES}

In this Appendix, we give a brief description of the three measures of spike train synchrony [30] that we use in this paper: the time-scale-dependent van Rossum distance [35] and the time-scale-independent ISI and SPIKE distances in their adaptive generalizations $[36,37,42]$.

\section{1. van Rossum spike train distance}

For the van Rossum distance, each spike time $t_{n}$ of both spike trains $\left(n=1, \ldots, N_{s}\right.$, where $N_{s}$ is the total number of spikes in the point process) is convolved with an exponential kernel

$$
e^{-\left(t-t_{n}\right) / \tau_{R}} \quad\left(t>t_{n}\right) .
$$

The parameter of this measure is $\tau_{R}$. The convolved waveforms are called $f_{1}(t)$ for the first and $f_{2}(t)$ for the second spike train. The van Rossum distance $D_{R}[35]$ is then calculated as

$$
D_{R}\left(\tau_{R}\right)=\frac{1}{\tau_{R}} \int_{0}^{\infty}\left[f_{1}(t)-f_{2}(t)\right]^{2} d t .
$$

For high and low $\tau_{R}$, the distance acts as a spike rate and spike timing measure, respectively.

\section{ISI and SPIKE distances}

Both the ISI and the SPIKE distances compute first a time-resolved spike dissimilarity profile. The ISI-dissimilarity profile $I(t)$ is based on the interspike intervals, whereas the SPIKE-dissimilarity profile $S(t)$ takes into account the exact timing of the spikes. For both measures, the total distance between two spike trains is then calculated as an integration over time of the instantaneous spike dissimilarity profile:

$$
D_{P}=\frac{1}{t_{e}-t_{s}} \int_{t_{s}}^{t_{e}} P(t) d t, \quad P=I, S,
$$

where $t_{s}$ and $t_{e}$ are the beginning and the end of the interval.

In the calculation of the dissimilarity profiles, there is always an ambiguity concerning the interval preceding the very first and following the very last spike. In our case, we initially calculate the profile for the whole time series, and afterward we segment it in overlapping windows and compute the integrals. This way, for the intermediate windows we use some information about the spikes in neighboring windows, and the problem remains only at the very beginning and the very end of the spike trains. For this reason, we ignore the first and the last $W_{\mathrm{ex}}$ windows of our signals. The value of $W_{\mathrm{ex}}$ is set adaptively as the minimum number of windows that we have to neglect in order not to consider windows with empty neighbor windows.

Recently adaptive generalizations of the ISI and the SPIKE distances were proposed [42], which take into account the relative importance of local differences compared to the global time scale. By relying on a minimal relevant time scale, they basically give less importance to the differences that are smaller than a threshold $\mathcal{T}$. This threshold is computed as the mean of the second moments of the ISIs of the whole spike train. It is important to calculate $\mathcal{T}$ for each spike train from the whole signal and not just from individual windows in order to compute a meaningful distance between different windows of the same signal. In contrast, $\mathcal{T}$ can in general be different for the two spike trains whose connection is under investigation. In fact, the measure $L$ evaluates how the similarity between different windows of one signal is mapped on the similarity among different windows of the other signal.

In this paper, we only show the values of $L$ computed with the adaptive generalization of ISI and SPIKE distances. Their property of taking into account the global information about the time scale and not only adapting to the local one is always desirable [42]. Additionally, in particular for $L_{\text {SPIKE }}$ this is also reflected in a higher robustness to noise. Here we briefly describe how to compute these two dissimilarities profiles, using the notation of Mulansky et al. [60].

\section{a. ISI distance}

The ISI-dissimilarity profile $I(t)$ is based on the relative length of simultaneous interspike intervals [36]. For every time $t$, let $t_{P}^{(1),(2)}(t)$ be the time of the last preceding spike, and let $t_{F}^{(1),(2)}(t)$ be the time of the first following spike, for the first and the second spike train, respectively. The instantaneous interspike interval is thus defined as $v^{(1)}(t)=t_{F}^{(1)}-t_{P}^{(1)}$, and analogously for $v^{(2)}(t)$. The profile $I(t)$ is computed by normalizing the absolute difference of the interspike intervals:

$$
I(t)=\frac{\left|v^{(1)}(t)-v^{(2)}(t)\right|}{\max \left\{v^{(1)}(t), v^{(2)}(t)\right\}} .
$$

By construction, $v^{(1)}(t)$ and $v^{(2)}(t)$ are piecewise constant functions, and the same holds for $I(t)$. The adaptive generalization [42] takes into account the threshold value $\mathcal{T}$ :

$$
I^{A}(t)=\frac{\left|v^{(1)}(t)-v^{(2)}(t)\right|}{\max \left\{v^{(1)}(t), v^{(2)}(t), \mathcal{T}\right\}} .
$$

\section{b. SPIKE distance}

The SPIKE-dissimilarity profile focuses on the exact timing of spikes. For every time $t$, the distance from the last preceding spike to the closest spike of the other spike train is defined as

$$
\Delta t_{P}^{(1)}(t)=\min _{i}\left\{\left|t_{p}^{(1)}-t_{i}^{(2)}\right|\right\}
$$

and analogously for $\Delta t_{P}^{(2)}$ and $\Delta t_{F}^{(1),(2)}$. Then these values are weighted by the instantaneous distances to the two nearest spikes:

$$
\begin{aligned}
& x_{P}^{(1)}(t)=t-t_{P}^{(1)}, \\
& x_{F}^{(1)}(t)=t_{F}^{(1)}-t
\end{aligned}
$$

with $x_{P}^{(1)}(t)+x_{F}^{(1)}(t)=v^{(1)}(t)$. In this way, the local dissimilarity is obtained as

$$
S_{1}(t)=\frac{\Delta t_{P}^{(1)}(t) x_{P}^{(1)}(t)+\Delta t_{F}^{(1)}(t) x_{F}^{(1)}(t)}{v^{(1)}(t)},
$$

and $S_{2}(t)$ is defined analogously. 
The SPIKE-dissimilarity profile $S(t)$ is obtained from these local distances $S_{1}(t)$ and $S_{2}(t)$, weighted by the local interspike intervals, with a final normalization:

$$
S(t)=\frac{S_{1}(t) v^{(2)}(t)+S_{2}(t) v^{(1)}(t)}{2\left\langle v^{(1,2)}(t)\right\rangle^{2}} .
$$

Notice that $S(t)$ is a piecewise linear function. The adaptive generalization in this case is

$$
S^{A}(t)=\frac{S_{1}(t) v^{(2)}(t)+S_{2}(t) v^{(1)}(t)}{2\left\langle v^{(1,2)}(t)\right\rangle \max \left\{\left\langle v^{(1,2)}(t)\right\rangle, \mathcal{T}\right\}} .
$$

[1] K. J. Friston, Brain Connect. 1, 13 (2011).

[2] C. J. Stam, Clin. Neurophysiol. 116, 2266 (2005).

[3] E. Pereda, R. Q. Quiroga, and J. Bhattacharya, Prog. Neurobiol. 77, 1 (2005).

[4] R. E. Greenblatt, M. Pflieger, and A. Ossadtchi, J. Neurosci. Methods 207, 1 (2012).

[5] M. S. Lewicki, Network 9, R53 (1998).

[6] R. E. Kass, V. Ventura, and E. N. Brown, J. Neurophysiol. 94, 8 (2005).

[7] A. M. Aertsen and G. L. Gerstein, Brain Res. 340, 341 (1985).

[8] S. Grün, J. Neurophysiol. 101, 1126 (2009).

[9] R. Dzakpasu and M. Żochowski, Physica D 208, 115 (2005).

[10] Z. Li, G. Ouyang, D. Li, and X. Li, Phys. Rev. E 84, 021929 (2011).

[11] B. Gourévitch and J. J. Eggermont, J. Neurophysiol. 97, 2533 (2007).

[12] O. Stetter, D. Battaglia, J. Soriano, and T. Geisel, PLoS. Comput. Biol. 8, e1002653 (2012).

[13] A. G. Nedungadi, G. Rangarajan, N. Jain, and M. Ding, J. Comput. Neurosci. 27, 55 (2009).

[14] M. Krumin and S. Shoham, Comput. Intell. Neurosci. 2010 , 752428 (2010).

[15] I. H. Stevenson, J. M. Rebesco, L. E. Miller, and K. P. Körding, Curr. Opin. Neurobiol. 18, 582 (2008).

[16] F. Van Bussel, B. Kriener, and M. Timme, Front. Comput. Neurosci. 5, 3 (2011).

[17] A. Pikovsky, Phys. Rev. E 93, 062313 (2016).

[18] R. Cestnik and M. Rosenblum, Phys. Rev. E 96, 012209 (2017).

[19] A. Čenys, G. Lasiene, and K. Pyragas, Physica D 52, 332 (1991).

[20] S. J. Schiff, P. So, T. Chang, R. E. Burke, and T. Sauer, Phys. Rev. E 54, 6708 (1996).

[21] J. Arnhold, P. Grassberger, K. Lehnertz, and C. E. Elger, Physica D 134, 419 (1999).

[22] R. Q. Quiroga, J. Arnhold, and P. Grassberger, Phys. Rev. E 61, 5142 (2000).

[23] D. Chicharro and R. G. Andrzejak, Phys. Rev. E 80, 026217 (2009).

[24] J. J. González, L. D. Méndez, S. Mañas, M. R. Duque, E. Pereda, and L. De Vera, Clin. Neurophysiol. 124, 1139 (2013).

[25] G. Alba, E. Pereda, S. Mañas, L. D. Méndez, M. R. Duque, A. González, and J. J. González, Clin. Neurophysiol. 127, 1321 (2016).

[26] R. Rial, J. González, L. Gené, M. Akaârir, S. Esteban, A. Gamundí, P. Barceló, and C. Nicolau, Am. J. Physiol. Regul. Integr. Comp. Physiol. 304, R232 (2013).

[27] P. Papiotis, M. Maestre, M. Marchini, and A. Perez, Synchronization of intonation adjustments in violin duets: towards an objective evaluation of musical interaction (unpublished).

[28] R. G. Andrzejak and T. Kreuz, Europhys. Lett. 96, 50012 (2011).

[29] T. Sauer, Phys. Rev. Lett. 72, 3811 (1994).

[30] T. Kreuz, Scholarpedia 6, 11934 (2011).

[31] R. G. Andrzejak, D. Chicharro, K. Lehnertz, and F. Mormann, Phys. Rev. E 83, 046203 (2011).
[32] M. Paluš and M. Vejmelka, Phys. Rev. E 75, 056211 (2007).

[33] R. G. Andrzejak, A. Kraskov, H. Stögbauer, F. Mormann, and T. Kreuz, Phys. Rev. E 68, 066202 (2003).

[34] J. D. Victor and K. P. Purpura, J. Neurophysiol. 76, 1310 (1996).

[35] M. C. van Rossum, Neural Comput. 13, 751 (2001).

[36] T. Kreuz, J. S. Haas, A. Morelli, H. D. I. Abarbanel, and A. Politi, J. Neurosci. Methods 165, 151 (2007).

[37] T. Kreuz, D. Chicharro, C. Houghton, R. G. Andrzejak, and F. Mormann, J. Neurophysiol. 109, 1457 (2013).

[38] R. Narayan, G. Graña, and K. Sen, J. Neurophysiol. 96, 252 (2006).

[39] J. Goulet, J. L. van Hemmen, S. N. Jung, B. P. Chagnaud, B. Scholze, and J. Engelmann, J. Neurophysiol. 107, 2581 (2012).

[40] T. Bockhorst and U. Homberg, J. Neurophysiol. 113, 3291 (2015).

[41] L. Zapata-Fonseca, D. Dotov, R. Fossion, and T. Froese, Front. Psychol. 7, 1940 (2016).

[42] E. Satuvuori, M. Mulansky, N. Bozanic, I. Malvestio, F. Zeldenrust, K. Lenk, and T. Kreuz, J. Neurosci. Methods 287, 25 (2017).

[43] J. Theiler, Phys. Rev. A 34, 2427 (1986).

[44] A. Kaiser and T. Schreiber, Int. J. Bifurcation Chaos 14, 1987 (2004).

[45] R. D. Pinto, P. Varona, A. R. Volkovskii, A. Szücs, H. D. I. Abarbanel, and M. I. Rabinovich, Phys. Rev. E 62, 2644 (2000).

[46] J. P. Shaffer, Annu. Rev. Psychol. 46, 561 (1995).

[47] N. F. Rulkov, M. M. Sushchik, L. S. Tsimring, and H. D. I. Abarbanel, Phys. Rev. E 51, 980 (1995)

[48] L. Kocarev and U. Parlitz, Phys. Rev. Lett. 76, 1816 (1996).

[49] H. D. I. Abarbanel, N. F. Rulkov, and M. M. Sushchik, Phys. Rev. E 53, 4528 (1996).

[50] G. B. Ermentrout, R. F. Galán, and N. N. Urban, Trends Neurosci. 31, 428 (2008).

[51] N. Kasabov, Neural Netw. 23, 16 (2010).

[52] M. G. Rosenblum and A. S. Pikovsky, Phys. Rev. E 64, 045202 (2001).

[53] D. A. Smirnov and R. G. Andrzejak, Phys. Rev. E 71, 036207 (2005).

[54] P. Laiou and R. G. Andrzejak, Phys. Rev. E 95, 012210 (2017).

[55] R. G. Andrzejak, F. Mormann, and T. Kreuz, Phys. Rev. E 90, 062906 (2014).

[56] N. Boers, B. Bookhagen, H. M. J. Barbosa, N. Marwan, J. Kurths, and J. A. Marengo, Nat. Commun. 5, 5199 (2014).

[57] T. Kreuz, E. Satuvuori, M. Pofahl, and M. Mulansky, New J. Phys. 19, 043028 (2017).

[58] Y. Hirata, K. Iwayama, and K. Aihara, Phys. Rev. E 94, 042217 (2016).

[59] http://ntsa.upf.edu/downloads, http://www.fi.isc.cnr.it/users/ thomas.kreuz/sourcecode.html

[60] M. Mulansky, N. Bozanic, A. Sburlea, and T. Kreuz, in International Conference on EBCCSP (IEEE, Piscataway, NJ, 2015), pp. 1-8. 